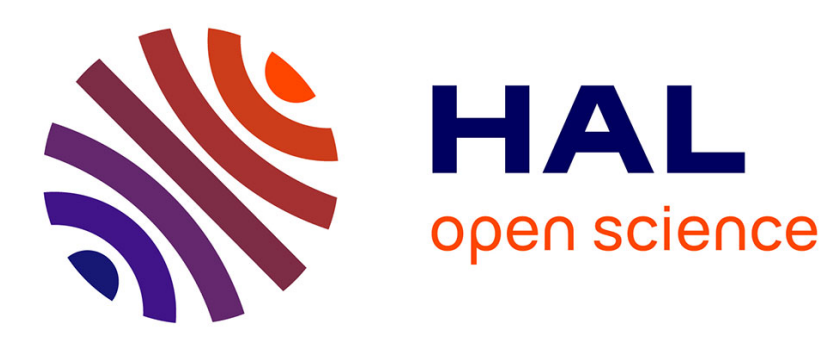

\title{
Le nouveau climat des expéditions naturalistes au sud
} Elsa Faugere, Selim Louafi

\section{To cite this version:}

Elsa Faugere, Selim Louafi. Le nouveau climat des expéditions naturalistes au sud. Revue Tiers Monde, 2011, 207 (3), 10.3917/rtm.207.0079 . halshs-01664802

\section{HAL Id: halshs-01664802 https://shs.hal.science/halshs-01664802}

Submitted on 15 Dec 2017

HAL is a multi-disciplinary open access archive for the deposit and dissemination of scientific research documents, whether they are published or not. The documents may come from teaching and research institutions in France or abroad, or from public or private research centers.
L'archive ouverte pluridisciplinaire HAL, est destinée au dépôt et à la diffusion de documents scientifiques de niveau recherche, publiés ou non, émanant des établissements d'enseignement et de recherche français ou étrangers, des laboratoires publics ou privés. 


\section{LE NOUVEAU CLIMAT DES EXPÉDITIONS NATURALISTES AU SUD L'exemple de santo 2006 au vanuatu \\ Elsa Faugère, Sélim Louafi}

\section{Armand Colin | « Revue Tiers Monde »}

2011/3 n²07 | pages 79 à 96

ISSN 1293-8882

ISBN 9782200927325

Article disponible en ligne à l'adresse :

https://www.cairn.info/revue-tiers-monde-2011-3-page-79.htm

\section{Pour citer cet article :}

Elsa Faugère, Sélim Louafi « Le nouveau climat des expéditions naturalistes au sud. L'exemple de santo 2006 au vanuatu », Revue Tiers Monde 2011/3 (n²07), p. 79-96. DOI 10.3917/rtm.207.0079

Distribution électronique Cairn.info pour Armand Colin.

(C) Armand Colin. Tous droits réservés pour tous pays.

La reproduction ou représentation de cet article, notamment par photocopie, n'est autorisée que dans les limites des conditions générales d'utilisation du site ou, le cas échéant, des conditions générales de la licence souscrite par votre établissement. Toute autre reproduction ou représentation, en tout ou partie, sous quelque forme et de quelque manière que ce soit, est interdite sauf accord préalable et écrit de l'éditeur, en dehors des cas prévus par la législation en vigueur en France. Il est précisé que son stockage dans une base de données est également interdit. 


\title{
LE NOUVEAU CLIMAT DES EXPÉDITIONS NATURALISTES AU SUD
}

\author{
L'exemple de Santo 2006 au Vanuatu*
}

\author{
Elsa Faugère $^{\star \star}$, Sélim Louafi ${ }^{\star \star \star}$
}

À partir de tensions apparues lors du montage de l'expédition Santo 2006 entre certains membres du gouvernement du Vanuatu et les organisateurs français de cette expédition, nous analyserons les liens entre sciences et sociétés dont témoigne la politique culturelle mise en place par ce pays. La notion de Consentement informé préalable, l'apparition d'une " nouvelle question indigène » et les débats internationaux actuels en matière de bioprospection fournissent un arrière-plan complexe dans lequel prennent sens les tensions évoquées.

Mots clés : Expédition, collectes, naturalistes, Vanuatu, consentement informé, bioprospection.

L'ambiance était tendue, crispée, le bureau, petit et sombre. Notre interlocutrice, membre des services de l'environnement du gouvernement du Vanuatu, donna immédiatement le ton : «Vous devez laisser un double des spécimens collectés au Vanuatu ; vous devez obtenir le consentement préalable des populations locales avant de collecter leurs savoirs ; et vous devez passer par le Centre culturel du Vanuatu pour tout ce qui concerne le module « Diversité culturelle».

L’organisateur de l'Expédition Santo 2006 s'emporta pour la première fois. " On est obligé », lui répondit-il, « de tout emporter parce que $98 \%$ des espèces qui seront collectées sont inconnues. Et ce sont des spécialistes du monde entier qui feront ce long travail d'identification qui mettra des mois, voire des années,

\footnotetext{
* Nous remercions les trois organisateurs de l'expédition, Philippe Bouchet, Olivier Pascal et Hervé Le Guyader, pour la confiance qu'ils nous ont témoignée, ainsi que tous les participants pour leur disponibilité à notre égard. L'analyse de cette expédition s'inscrit dans le cadre d'un projet de recherche financé, depuis janvier 2010, par l'Agence nationale de la recherche, intitulé " Expébiodiv. Étude pluridisciplinaire des expéditions naturalistes contemporaines » et coordonné par Elsa Faugère.

** INRA, Ecodéveloppement, faugere@avignon.inra.fr

*** CIRAD, UMR AGAP, louafi@cirad.fr
} 
avant d'aboutir. Quant au Centre culturel, nous ne savions pas qu'il jouait un rôle aussi déterminant, mais nous allons réparer cet oubli ».

Voilà en substance le contenu de cette discussion tendue qui s'est déroulée en novembre 2005 entre les organisateurs de l'Expédition Santo 2006 et cette représentante du gouvernement du Vanuatu, à propos de la Convention (Memorandum of Understanding) que ces deux parties devaient signer.

De telles demandes montrent, selon les organisateurs, à quel point leurs interlocuteurs sont mal informés de la nature de leurs recherches taxonomiques. Comment obtenir le consentement informé préalable des populations locales lorsqu'on collecte des micro-organismes marins invisibles à l'œil nu ? Comment laisser un double systématique des échantillons alors qu'en matière zoologique, on ne sait pas ce qu'on récolte et qu'il faudra des années pour le savoir? Et comment le Vanuatu, l'un des pays les plus pauvres du monde selon les indicateurs de l'ONU, pourrait-il conserver des dizaines de milliers de spécimens, étant donné les difficultés et les coûts de cette conservation ex situ?

Nous étions bien au cœur des difficultés posées par certains articles de la Convention sur la diversité biologique (CDB, 1992), signée en 1992 et entrée en vigueur le 29 décembre 1993. Depuis lors, l'accès à la biodiversité des pays mégadivers par les scientifiques a été profondément modifié. Pourtant, les organisateurs de l'Expédition Santo 2006 ont eu comme souci de respecter l'esprit de la $\mathrm{CDB}$, notamment en termes d'accès à la biodiversité et de « partage des avantages » lié à son utilisation. C'était d'ailleurs l'un des objectifs de cette mission préparatoire.

Les tensions qui sont apparues au cours de cette entrevue et les conséquences qu'elles ont eues sur le module ethnologique de l'Expédition Santo 2006 nous paraissent exemplaires des rapports actuels entre sciences et sociétés. C'est de cela qu'il va être question dans cet article. Il est en effet particulièrement significatif que l'une des principales pommes de discorde, au cours du montage de cette grande expédition naturaliste, concerne un module somme toute marginal, puisqu'il s'agissait du module « Diversité culturelle ». C'était d'ailleurs l'une des originalités de cette expédition : inclure, aux côtés des quatre modules naturalistes, un module ethnologique. Mais, à la suite de cette discussion et d'autres échanges qui s'ensuivirent entre les organisateurs et des membres du gouvernement du Vanuatu, le contenu de ce module a profondément changé et ce à tel point qu'il a même disparu de l'organisation de l'expédition en tant que module autonome.

Organisée par le Muséum national d'histoire naturelle de Paris, l'Institut de recherche pour le développement et l'ONG ProNatura International, l'expédition Santo a réuni 160 scientifiques d'une vingtaine de pays différents d'août à décembre 2006. Financé majoritairement par des fondations (d'entreprises et 
familiales), principalement la fondation Niarchos et la fondation Total, le budget de l'expédition (hors salaires des participants) s'élevait à 1,2 million d'euros. L'expédition était organisée en quatre modules naturalistes couvrant la diversité des milieux de l'île de Santo :

- le module marin (organisé par Philippe Bouchet, professeur au MNHN, malacologue) ;

- le module forêts montagnes rivières (organisé par Bruno Corbara, directeur scientifique du Radeau des cimes, Université de Clermont Ferrand, entomologiste, et Philippe Keith, professeur au MNHN, ichtyologue) ;

- le module karst (organisé par Louis de Harveng, directeur de recherche au CNRS, entomologiste) ;

- le module friches et aliens (organisé par Michel Pascal, directeur de recherche à l'INRA, écologue, spécialiste des espèces invasives).

Dans une première partie, nous analyserons le montage politique et administratif de l'expédition (la rédaction d'un Memorandum of Understanding) en partant des tensions évoquées ci-dessus. Ce faisant, nous procéderons à deux niveaux de contextualisation :

- par rapport à la politique culturelle mise en place dès le milieu des années 1970 par les Nouvelles Hébrides, et poursuivie par le Vanuatu indépendant en 1980 ;

- par rapport à la diffusion et à la généralisation, au cours des années 1990, de la notion de Consentement informé préalable dans le domaine des droits des populations indigènes et de leurs liens à la biodiversité.

Nous verrons alors que la politique culturelle du Vanuatu et la généralisation de la notion de Consentement informé préalable sont liées à ce que certains auteurs qualifient de "nouvelle question indigène ».

Dans une troisième partie, nous analyserons les conséquences scientifiques de ces tensions en retraçant les changements intervenus dans le module ethnologique et en les contextualisant par rapport au débat international en matière de bioprospection et de biopiraterie.

\section{« SCIENCE COUTUMIÈRE \# ET « NOUVELLE QUESTION INDIGĖNE \#}

À la suite de la conversation du début, les autorités du Vanuatu ont modifié la première version de la Convention et ajouté un long paragraphe, dans l'article 2 consacré aux " Obligations de l'expédition », où ils précisent les modalités de 
" recueil des savoirs traditionnels et indigènes » et les modalités d'utilisation qui pourront en être faites ${ }^{1}$ :

«Pour ce qui concerne la collecte des savoirs traditionnels et indigènes, le Groupe de Projet Santo s'engage à consulter le Centre culturel du Vanuatu, et à suivre les principes suivants : 1. Toute information émanant d'informateurs ni-Vanuatu et qui constitue un savoir traditionnel ou indigène ne devra être recueillie :

a) qu'après que les informateurs aient donné leur consentement préalable à fournir leurs informations pour les objectifs du projet ;

b) en précisant explicitement à qui cette information pourra être divulguée ; et

c) avec la mention explicite qu'il s'agit d'un savoir traditionnel ou indigène, et en l'attribuant à l'informateur, à son clan et à sa communauté.

2. La mise dans le domaine public (sous forme de publication ou sous une autre forme) de cette information sera fonction des réserves éventuellement émises par les informateurs (voir le point b ci-dessus). À cet effet, le Centre culturel devra donner son accord préalable à toute publication qui contiendrait des éléments de savoir traditionnel ou indigène.

3. Toute publication de cette information devra la situer dans son contexte et faire référence à l'informateur (voir le point c ci-dessus).

4. Des doubles de toute information constituant un savoir traditionnel ou indigène devront être déposés au Centre culturel du Vanuatu sous forme brute (non mise en forme) dans l'état où elle a été recueillie (document audio, vidéo, photographique, écrit ou imprimé) avec indication complète de l'origine » (Extrait du Memorandum of Understanding, 2005).

Par cet article, le gouvernement du Vanuatu révèle, d'une part, le rôle central que joue le Centre culturel en matière de recherche sur les « savoirs traditionnels et indigènes » et, d'autre part, son adhésion à un vaste mouvement international que certains qualifient de « nouvelle question indigène » (Fritz, Deroche, Fritz, Porteilla, 2005).

\section{Le Centre culturel dans I'histoire politique du Vanuatu}

Le Centre culturel du Vanuatu apparaît donc comme un partenaire incontournable pour qui veut travailler sur les « savoirs traditionnels et indigènes ». Or, les organisateurs de l'expédition n'avaient pas pris conscience de l'importance de ce Centre culturel dans l'accès, non pas à la biodiversité, mais bien aux « savoirs locaux » en général et à ceux portant sur la biodiversité en particulier.

L'importance du Centre culturel en matière de savoirs considérés comme traditionnels remonte au milieu des années $1970^{2}$. À cette époque du condominium franco-britannique, un linguiste français, Jean-Michel Charpentier, lance un programme intitulé « Tradition orale ». Il avait comme objectif d'enregistrer les traditions orales de ce qui était encore les Nouvelles Hébrides. Kirk Huffman,

\footnotetext{
1. Pour être tout à fait précis, la notion de Consentement informé préalable était présente dans la version initiale de la Convention, mais seulement dans l'article 2 qui précisait les obligations des chercheurs en matière de collectes naturalistes. II était ainsi écrit : les chercheurs « s'engagent à n'accéder au terrain et à ne récolter des échantillons qu'après avoir obtenu l'accord préalable des propriétaires coutumiers et autres parties concernées ». Une distinction est ainsi faite entre deux types de collectes : une collecte naturaliste d'échantillons de flore et de faune, et une collecte ethnologique de savoirs traditionnels et indigènes.
}

2. Pour retracer l'histoire de cette politique, nous avons utilisé deux articles : Bolton (1999) et Tryon (1999). 
conservateur du Centre culturel de 1977 à 1990, alors jeune anthropologue britannique, soutiendra cette initiative et fera diffuser ces enregistrements à la radio dans le but de participer à la préservation des «traditions culturelles». Avec l'indépendance du Vanuatu en 1980, il poursuivit ce programme d'enregistrement, de collecte et d'archivage des traditions locales, en créant un réseau d'enquêteurs de terrains, les fieldworkers. Membres considérés comme importants dans leur " communauté » d'origine, ces fieldworkers seront chargés d'effectuer ces collectes et ces enregistrements. Tous les ans, grâce à une subvention du gouvernement australien provenant du Fond des cultures du Pacifique Sud, ils suivent une formation aux techniques d'enquête qui leur est délivrée par un linguiste australien, Darell Tryon. Ces enregistrements sont conservés dans une salle spéciale du Centre culturel, appelé Tabu Room, et dont l'accès est strictement réglementé. Selon Darell Tryon que nous avons rencontré en novembre 2005, l'objectif de cette politique de recueil et d'archivage de la " coutume" (kastom) est de constituer une "science coutumière ", ou " science mélanésienne ».

Entre 1985 et 1994, le gouvernement du Vanuatu instaure un moratoire dans son pays, interdisant toute recherche en sciences humaines menée par des chercheurs étrangers (Bolton, 1999, p. 4). Seule l'entreprise des fieldworkers se poursuivra pendant cette période. Selon Lissant Bolton (idem, p. 4), ce moratoire était l'expression d'une identité nationale que le gouvernement de Walter Lini cherchait à affirmer dans ces premières années de l'indépendance. La kastom, dénigrée et dévalorisée par les missionnaires et les colons pendant la période coloniale, devenait le symbole de l'identité nationale et la propriété des ni-Vanuatu. Seuls ces derniers pouvaient et devaient l'étudier. Elle ne pouvait pas devenir un objet d'étude pour des chercheurs étrangers.

En 1994, le moratoire est levé. Les recherches en sciences humaines reprennent dans le pays. Mais elles doivent dès lors se faire en étroite collaboration avec les fieldworkers qui décident, ou refusent, d'attribuer les permis de recherche sur la base de l'intérêt de la recherche pour les populations locales. De plus, ces recherches doivent se faire sous la forme d'une véritable collaboration entre chercheurs et ni-Vanuatu : les produits de la recherche sont considérés comme des co-productions entre savoirs scientifiques et « savoirs traditionnels et locaux ».

Il convient de noter les similitudes entre ce choix fait par le gouvernement du Vanuatu, dès le début des années 1980, et les expressions de "science citoyenne ", aujourd'hui utilisée en France et popularisée par des scientifiques américains dans les années 1990, dans le cadre d'un programme d'observation des oiseaux de jardins, appelé Feederwatch, qui associe étroitement « profanes » et scientifiques (Charvolin, 2004). 


\section{La " nouvelle question indigène "}

Dans les années 1980, et plus encore dans les années 1990, la question des populations indigènes prit une ampleur sans précédent dans les arènes internationales. La politique de recherche mise en place au Vanuatu s'inscrit dans ce vaste mouvement qui s'effectue de manière concomitante dans trois arènes distinctes : au sein de l'Organisation des Nations unies, au sein de la communauté scientifique des ethnobiologistes et au sein d'un réseau d'ONG autochtones, actives dès les années 1970 en Amérique du Nord.

Débutée dans les années 1980, l'internationalisation de la question autochtone répond à deux motivations : l'incapacité de ces populations à obtenir justice des gouvernements des États dont ils dépendent et leur refus de se reconnaître dans les catégories politiques existantes (Brahy, Louafi, 2003).

L'une des étapes les plus importantes est la création en 1982 d'un Groupe de travail des populations autochtones au sein de la Commission des Droits de l'Homme des Nations unies. Selon Frédéric Deroche (2006, p. 68), « la création de ce groupe de travail représente un événement majeur pour la lutte des peuples autochtones car ils possèdent désormais une instance internationale où ils peuvent faire valoir leurs droits ; il symbolise l'ouverture des Nations unies à la cause autochtone ».

En 1988, lors du premier congrès de la Société internationale d'ethnobiologie, un groupe d'ethnobiologistes met également la question indigène sur l'agenda international par leur fameuse "Déclaration de Belém »3 (Dumoulin, 2003; Posey, Dutfield, 1997). Par cette déclaration, ces chercheurs veulent alerter la communauté internationale sur le constat d'une double extinction : de la diversité biologique et de la diversité culturelle. Tout en faisant un lien qu'ils considèrent comme « inextricable » entre diversité biologique et culturelle, ils veulent que "des mécanismes soient établis afin que les peuples autochtones soient indemnisés pour l'utilisation qui est faite de leurs connaissances et de leurs ressources biologiques ».

En 1990, lors de leur deuxième Congrès mondial, les ethnobiologistes créent " la Coalition mondiale pour la diversité bioculturelle». Cette coalition se donne comme objectif « d'amener les peuples autochtones, les organisations scientifiques et les groupes écologistes à travailler ensemble à la mise en œuvre d'une stratégie énergique portant sur l'utilisation des connaissances traditionnelles, la participation des populations locales à la réalisation des stratégies de conservation et de développement, et l'application de nouveaux modèles de conservation axés sur les populations » (Posey, Dutfield, 1997). 
En 1992, au Sommet de la Terre de Rio, où sera signée la Convention sur la diversité biologique, "cette coalition est soutenue par d'autres groupes, en particulier des ONG, comme l'Union mondiale pour la nature (UICN), Rural Advancement Foundation International (RAFI) et World Wild Fund (WWF), qui s'insurgent contre le biopiratage des ressources naturelles du Sud par les grandes firmes du Nord. Le début des années 1990 marque en effet le début de l'explosion des dépôts de brevets sur les semences et les produits issus de biotechnologies, d'une part, et le début des négociations du Gatt sur les droits de propriété intellectuelle, d'autre part. Cette coalition mondiale va alors porter le problème des savoirs traditionnels sur la scène internationale des négociations de Rio dans le cadre des droits de propriété intellectuelle (DPI) en jeu dans l'utilisation de la biodiversité » (IDDRI, 2002).

Faisant l'historique de l'émergence du mouvement international des peuples autochtones au XX siècle, Frédéric Deroche (2006) écrit : « La Conférence des Nations unies sur l'environnement et le développement de Rio permet aux peuples autochtones de prendre part pour la première fois en séance plénière : le droit à la parole en tant que " peuple autochtone » leur est accordé. Elle reconnaît leur rôle essentiel dans la gestion de l'environnement et du développement en raison de leur savoir et de leurs pratiques traditionnelles " (idem, 2006, p. 70) ${ }^{4}$.

Dans les années 1990, ce mouvement s'accélère au niveau international, avec notamment la mise en place d'une décennie internationale (1995-2004) des peuples autochtones.

Au cours de la seconde moitié des années 1990, la question des savoirs indigènes, et notamment de leurs liens avec les droits de propriété intellectuelle, sera l'objet d'intenses débats internationaux au sein de la CDB, mais aussi de l'Organisation mondiale pour la propriété intellectuelle (OMPI) et de l'Organisation mondiale du commerce (OMC).

Sans entrer davantage dans le détail de cette histoire, il nous importe ici de montrer que le Vanuatu s'inscrit pleinement dans ce mouvement international qui, d'une part, a ouvert la possibilité d'une marchandisation des «savoirs indigènes » en les liant aux droits de propriété intellectuelle et qui, d'autre part, vise à instaurer des collaborations et des partenariats beaucoup plus étroits entre chercheurs et "communautés indigènes et locales ". Il s'agit là de donner des marges de manœuvre mais aussi, et surtout, de contrôle aux populations locales et indigènes, en matière de recherche scientifique. La notion de Consentement informé préalable (CIP), mentionnée dans la Convention signée entre les organisateurs de l'Expédition Santo 2006 et le Gouvernement du Vanuatu, en constitue le symbole majeur.

4. Ces questions constitueront le cœur du fameux article $8 \mathrm{~J}$ de la CDB. 


\section{LE CONSENTEMENT INFORMÉ PRÉALABLE : ÉLÉMENTS D'HISTOIRE ET SIGNIFICATIONS SOCIALES}

Même si les organisateurs de Santo 2006 avaient bien mentionné, dans leur première version du Memorandum Of Understanding, la nécessité d'obtenir le Consentement informé préalable « des propriétaires coutumiers et autres parties concernées » avant toute récolte d'échantillons de flore et de faune, ils étaient plutôt perplexes et critiques par rapport à cette notion. Ils se demandaient comment obtenir le Consentement informé préalable des populations locales en collectant des organismes vivants qui ne font pas sens pour ces populations (ces organismes étant invisibles ou inconnus d'eux). Et ils remarquaient que cette procédure, impulsée par la CDB en matière d'accès à la biodiversité, peut non seulement alourdir et compliquer considérablement leurs recherches ou, pire, leur interdire carrément l'accès à la biodiversité dans certains pays. Ils considèrent ainsi qu'une telle procédure est inadaptée à la recherche taxonomique fondamentale qui a comme seul objectif de produire un savoir scientifique sur la nature.

Avant d'en venir à une analyse de la notion de Consentement informé préalable en matière d'accès à la biodiversité (aux ressources biologiques et aux « savoirs traditionnels »), une mise en perspective historique de cette notion nous paraît nécessaire.

\section{Le CIP dans le domaine médical}

La notion de Consentement informé est fondée sur des principes éthiques selon lesquels il faut respecter la dignité et la valeur de tous les êtres humains et leur droit à l'autodétermination (Miller, Boulton, 2007). C'est le code de Nuremberg, rédigé en 1947 en réponse aux atrocités des expériences nazies sur des sujets humains, qui va en faire une notion centrale pour des pratiques de recherche éthiques (Gray, 1978).

Comme le soulignent Tina Miller et Mary Boulton (2007), si le Code de Nuremberg n'était pas le premier à fixer des principes éthiques pour la recherche, il sera cependant le plus influent. C'est en effet lui qui va directement influencer la Déclaration universelle des Droits de l'Homme (1948) et la Déclaration d'Helsinki (1964). Le Consentement informé est alors institué comme le principal mécanisme pour protéger les participants d'une recherche contre toute exploitation et dommage (Miller, Boulton, 2007). La Déclaration d'Helsinki, rédigée par l'Association médicale mondiale (World Medical Association), va faire du Consentement informé une exigence absolue pour la recherche médicale impliquant des participants humains, en déclarant que « les sujets doivent être volontaires et informés » (idem, 2007). 
Dans les années 1970, l'exigence d'un Consentement informé s'étend à la pratique médicale elle-même, en raison, notamment, des progrès technologiques alors réalisés dans ce domaine. Développement de la bioéthique et progrès technologiques sont ainsi étroitement liés. Mais, dans ces années-là, de nombreuses critiques et réticences sont exprimées par les médecins à l'encontre du Consentement informé. Certains y voient une perte d'autorité et de pouvoir du médecin désormais tenu de dévoiler au patient sa connaissance médicale professionnelle. D'autres considèrent que le patient n'est pas équipé pour prendre des décisions médicales. Et enfin, le Consentement informé est vu par les médecins comme un obstacle majeur à l'avancée de la science (Gray, 1978, p. 40).

Dans les années 1970, la notion de Consentement informé est donc considérée comme menaçante pour la supériorité du savoir scientifique, médical en l'occurrence. En prétendant donner aux patients un rôle plus important dans les processus de décision, le Consentement informé conduirait à créer un modèle de soin plus égalitaire. Nous verrons plus loin que des travaux sociologiques récents remettent en question une telle vision.

\section{L'extension du Consentement informé préalable dans le champ des relations internationales}

La communauté internationale s'est emparée de la notion de Consentement informé préalable pour traiter de la gestion des risques liés à l'importation de substances chimiques et de pesticides. Face aux différences de capacités des pays à contrôler l'utilisation des substances chimiques dangereuses et des pesticides, les Nations unies ont développé un système de droit d'information sur les risques d'importation de telles substances.

Dans les années 1980, le programme des Nations unies pour l'environnement (PNUE) et la FAO (Organisation des Nations unies pour l'alimentation et l'agriculture) développent des lignes directrices relatives à l'échange d'information sur les substances chimiques et les pesticides prohibés ou d'usage sévèrement restreint. Ces lignes directrices constituent la base de ce qui deviendra la première procédure multilatérale de Consentement informé préalable.

Depuis l'entrée en vigueur de la Convention dite de Rotterdam en 2004, l'application de la procédure de Consentement préalable en connaissance de cause (CIP) est devenue une obligation juridiquement contraignante ${ }^{5}$.

\section{L'extension du Consentement informé préalable aux populations indigènes}

Dans les années 1980-1990, la notion de Consentement informé préalable va connaître une expansion aussi bien thématique que géographique.

5. http://www.pic.int/home_fr.php?type=t\&id=64\&sid $=41$ 
L'OIT (Organisation internationale du travail) est la première organisation intergouvernementale à se saisir de la question de la prise en compte des droits des peuples autochtones dans le processus de décision publique. Seul texte véritablement contraignant en vigueur, la convention 169 de l'OIT ${ }^{6}$ (1989) encourage des " procédures de codécision avec le gouvernement central » et de « participation de ces peuples aux mesures susceptibles d'avoir un retentissement sur leur existence » (Perrin, 2006, p. 269).

«Les peuples doivent avoir le droit de décider de leurs propres priorités en ce qui concerne le processus de développement [...] et d'exercer autant que possible un contrôle sur leur développement économique, social et culturel propre» (idem, 2006, p. 269).

Il faut donc consulter les populations concernées. Mais il ne s'agit alors que de consultation, il n'est pas encore question de consentement de leur part. «L'article 20 du projet de déclaration de 1994 des Nations unies sur les droits des peuples autochtones (qui n'a toujours pas été signé) est sur ce point plus exigeant quant aux modalités de participation de ces peuples aux décisions qui les concernent dans la mesure où il prévoit l'obligation pour les États d'obtenir le consentement, exprimé librement et en toute connaissance de cause, des peuples intéressés» (ibid., p. 272).

En matière de ressources naturelles et d'accès à la biodiversité, on trouve les premières traces de cette notion dans une série de rapports rédigés entre 1990 et 1994 par le Groupe de travail sur les populations autochtones de la Commission des Nations unies sur les corporations transnationales. Ce groupe de travail, chargé d'examiner les activités et les investissements des entreprises multinationales extractives (mines, pétrole, forêts, etc.) sur les territoires autochtones, constate l'existence d'alliances et de contrats entre les États et ces industries extractives, au détriment de l'intérêt des populations locales. Face à ces abus, ce groupe de travail élabore des procédures pour les protéger de l'exploitation de leurs ressources naturelles par certaines multinationales (cf. www.forestspeoples.org). C'est ainsi qu'il souligne l'importance de la participation des peuples autochtones dans les prises de décision concernant l'exploitation de leurs ressources naturelles. La notion de Consentement libre informé préalable apparaît alors. «Dans le droit international contemporain, les peuples autochtones ont le droit de participer aux prises de décision et de donner ou refuser leur consentement aux activités touchant à leurs terres, territoires et ressources ou à leurs droits en général » (cf. www.forestpeoples.org).

En matière d'accès à la biodiversité, la notion de Consentement informé préalable est présente dans la $\mathrm{CDB}$ ainsi que dans les décisions ultérieures de la Conférence des parties (COP), comme le notent deux anthropologues américains (Berlin, Berlin, 2003). Dans la CDB, elle est présente à deux endroits :

6. Convention 169 concernant les peuples indigènes et tribaux dans les pays indépendants. 
- explicitement dans l'article 15, concernant l'accès aux ressources génétiques qui est soumis au consentement préalable donné en connaissance de cause de la partie contractante qui fournit lesdites ressources ${ }^{7}$.

- et elle est également présente sous une autre appellation dans l'article 8j. sur la conservation in situ ${ }^{8}$. Il est ici question d'obtenir l'accord et la participation des populations autochtones et locales pour favoriser l'application sur une plus grande échelle de leurs connaissances, innovations et pratiques traditionnelles utiles à la conservation et à l'utilisation durable de la biodiversité.

Le groupe de travail ad hoc sur l'accès et le partage des avantages reconnaît que le CIP est l'élément fondamental pour toutes mesures d'accès aux ressources génétiques. Des principes servant de base aux procédures CIP sont énumérés :

- le requérant à l'accès à la biodiversité doit fournir suffisamment d'information pour obtenir le Consentement informé.

- le fournisseur doit être en mesure d'exiger si nécessaire plus d'information du requérant pour prendre sa décision.

- l'information fournie par le requérant doit être donnée dans un langage et d'une façon intelligible par le fournisseur.

Cette notion a enfin été consacrée au sein du récent Protocole de Nagoya, adopté en octobre 2010, qui porte sur l'accès aux ressources génétiques et le partage juste et équitable des avantages découlant de leur utilisation. Elle constitue une des rares revendications portées par les représentants des communautés autochtones et locales qui a été retenue au sein de ce Protocole.

\section{Le Consentement informé : un dispositif de libération individuelle et collective ou de pouvoir et de contrôle ?}

Le Consentement informé préalable des individus ou des « communautés locales » est généralement présenté comme un dispositif de protection et d'autonomisation d'acteurs considérés comme vulnérables (malades, populations autochtones et locales, etc.) contre des acteurs considérés comme dominants (médecin, multinationales, chercheurs, etc.). Dans le domaine de la recherche scientifique, l'obligation faite aux chercheurs d'obtenir le CIP avant toute collecte naturaliste et, de plus en plus, notamment en Amérique du Nord, avant toute enquête en sciences sociales, révèle une évolution des liens entre sciences et

\footnotetext{
7. Article 15 de la CDB (accès aux ressources génétiques), alinéa 5 : "l'accès aux ressources génétiques est soumis au consentement préalable donné en connaissance de cause de la Partie Contractante qui fournit lesdites ressources, sauf décision contraire de cette partie ».

8. Article 8 : Conservation in Situ : "Chaque partie contractante, dans la mesure du possible et selon qu'il conviendra : j. Sous réserve des dispositions de sa législation nationale, respecte, préserve et maintient les connaissances, innovations et pratiques des communautés autochtones et locales qui incarnent des modes de vie traditionnels présentant un intérêt pour la conservation et l'utilisation durable de la diversité biologique, et en favorise l'application sur une plus grande échelle, avec l'accord et la participation des dépositaires de ces connaissances, innovations et pratiques, et encourage le partage équitable des avantages découlant de l'utilisation de ces connaissances, innovations et pratiques ».
} 
sociétés qui va dans le sens d'une perte d'autonomie des scientifiques vis-à-vis du monde social. Est-ce que cette perte d'autonomie s'accompagne d'un processus d'autonomisation et de libération des individus ?

Il n'est pas possible de donner une réponse univoque à cette question complexe. Une étude sociologique récente (Ducournau, 2009) menée dans le champ de la santé apporte un éclairage intéressant. Cette étude montre notamment que, loin de conduire à plus d'autonomie individuelle, le consentement informé constitue un nouveau dispositif subtil d'exercice du pouvoir qui transfère la responsabilité de l'institution médicale aux patients eux-mêmes. Or, ces derniers ne souhaitent pas tous exercer cette responsabilité. Certains patients, dit l'auteur (idem, 2009, p. 314), « avaient pour particularité radicale de ne pas vouloir être acteur ni auteur de leur consentement éclairé. En accordant leur signature sans lecture des informations communiquées, ni non plus en posant des questions, ces participants placent de fait l'institution médicale et ses acteurs devant leurs propres responsabilités - morales - de dépositaires de la confiance. Au travers de ces «manières de faire » très proches de celles analysées par De Certeau, les acteurs donnent formellement l'impression de se soumettre à un dispositif puisqu'ils signent bien le formulaire de consentement éclairé mais, par leur attitude, ils le font "tourner à vide », et ils en exploitent l'ultime possibilité de s'en extraire et de le retourner paradoxalement contre lui-même : face à un dispositif institutionnel qui en appelle à l'action d'un sujet libre et informé, la marque de leur autonomie paraît être précisément de refuser ce statut du sujet pour mieux mettre en valeur la responsabilité institutionnelle elle-même ».

\section{QUELLE PLACE POUR LA RECHERCHE SCIENTIFIQUE DANS LE PROCESSUS DE VALORISATION DE LA BIODIVERSITÉ ?}

\section{Les aléas du module " ethnosciences " dans l'Expédition Santo $2006^{9}$}

En mars 2004, lors de la première réunion de lancement du projet Santo, un module appelé "Valorisation de la biodiversité » avait été envisagé. Ce module incluait non seulement un volet de bioprospection porté par un ethnopharmacologue de l'IRD (Institut de recherche sur le développement), mais aussi le développement de retombées pour l'écotourisme sur l'île de Santo. Le pré-programme scientifique rédigé en mai de la même année, reprenait ces deux idées dans un module intitulé « Développement durable / Valorisation de la biodiversité ». Tout en souhaitant inclure ces deux objectifs économiques, ce qui était original et novateur dans le cadre d'une expédition scientifique qui visait principalement à produire du savoir scientifique fondamental en inventoriant

9. Cf. Faugère (2008) pour une description détaillée des péripéties du module ethnosciences de l'expédition Santo. 
tous les compartiments de la biodiversité de l'île de Santo, les participants du projet Santo avaient, dès 2004, émis des craintes quant aux soupçons de biopiraterie dont ils pourraient être victimes. Jusqu'en décembre 2005, ce volet de bioprospection demeure pourtant dans le module ethnologique qui s'intitulait alors « Perceptions plurielles de la biodiversité ». À ce moment-là, il s'agissait de faire une comparaison des inventaires « savant » et "populaire » de la diversité biologique centrée sur la phyto-pharmacologie traditionnelle et les plantes tinctoriales. Pour ce faire, les ethno-écologues de ce module allaient devoir recueillir « les savoirs locaux sur la biodiversité ».

Et c'est précisément cet aspect qui a posé problème à notre interlocutrice du début, ainsi qu'à certains de ses collègues. Ils craignaient en effet que les ethnoécologues de l'expédition utilisent ces savoirs locaux à des fins commerciales, d'où leur demande d'obtenir préalablement le consentement informé des populations locales et de travailler en étroite collaboration avec le Centre culturel du Vanuatu. Pour les autorités du Vanuatu, l'objectif était là de mieux contrôler les recherches menées sur les savoirs locaux et leurs éventuelles retombées commerciales afin d'en négocier des contreparties.

Mais, face à ces réactions, les organisateurs de Santo ont préféré supprimer toute bioprospection, craignant, étant donné le climat international actuel sur le sujet, que cela ne mette en péril l'ensemble de l'expédition.

Pourtant, à la même période, le gouvernement du Vanuatu autorisait une biologiste allemande travaillant sur les connaissances médicinales " traditionnelles » du Vanuatu à conduire ses recherches. Celles-ci consistaient explicitement à tester les usages des plantes traditionnelles en termes de propriétés médicinales et pharmaceutiques. Il s'agissait bien pour elle de se baser sur les savoirs médicinaux traditionnels pour tenter de repérer des propriétés médicales et pharmaceutiques de ces plantes. L'objectif de ses recherches était donc clairement de la bioprospection. Le gouvernement du Vanuatu n'était donc pas hostile à la bioprospection en soi. La possibilité de bénéficier de retombées économiques et financières des recherches scientifiques l'intéressait fortement.

Étant donné le climat international très tendu sur ces questions de bioprospection et de biopiraterie, les organisateurs de Santo ont préféré ne pas prendre de risque et ne pas mettre en péril le cœur de l'Expédition qui était, rappelons-le, d'inventorier et d'archiver la biodiversité de cette île. Ils étaient bien avant tout dans une logique de production de connaissances académiques sur la nature, même s'ils étaient ouverts aux aspects de valorisation économique de la biodiversité et même s'ils tenaient à respecter l'esprit de la CDB. La valorisation économique de la biodiversité, dans la mesure où elle bénéficie aussi au pays fournisseur de biodiversité, est d'ailleurs pleinement dans l'esprit de la CDB. 
En dépit de la prudence des organisateurs de Santo 2006 qui les avait conduits à supprimer toute bioprospection, un article est paru le 28 juin 2006 dans le Daily Post, un des principaux quotidiens du Vanuatu (Royson, 2006). Dans cet article, le journaliste reprend les propos du directeur du Centre culturel du Vanuatu qui met en garde son gouvernement contre les dangers de biopiraterie liés à l'Expédition Santo 2006, comme, dit-il, à toute recherche scientifique naturaliste menée par des organismes étrangers au Vanuatu. Considérant que son pays ne dispose pas encore des moyens de contrôler efficacement de telles recherches, il propose d'y interdire toute recherche naturaliste, sur le modèle du moratoire de dix ans qui avait interdit, de 1984 à 1995, les recherches en sciences humaines. Pour appuyer sa démonstration, il prend l'exemple de brevets déposés par des chercheurs étrangers sur le kava, à partir des connaissances " traditionnelles » des populations locales et dont ces dernières ne tirent aucun bénéfice. Et il insiste sur le potentiel très lucratif des recherches scientifiques menées sur la biodiversité dont les populations locales peuvent attendre d'énormes bénéfices financiers.

Cet article sera sans conséquence sur la mise en œuvre de l'expédition Santo 2006, la convention ayant déjà été signée avec le gouvernement. Le directeur du Centre culturel voulait, semble-t-il, surtout profiter de ce contexte particulier pour faire passer ce message auprès de son gouvernement et le pousser à créer un Conseil national scientifique et un Conseil de la biodiversité qui serait chargé de mieux contrôler les recherches biologiques étrangères et de mieux en négocier les contreparties.

En effet, au-delà des rapports de force entre ministères et de la volonté du Centre culturel de peser davantage dans le débat national, l'expédition Santo 2006 ne prenait pas place dans un territoire vierge de tout débat national sur l'accès à la biodiversité. Port Vila avait accueilli, en avril 2001, un atelier national sur l'accès et le partage des avantages, ainsi qu'un Forum, en novembre 2002, sur l'établissement d'un Conseil national sur la recherche scientifique. Ce forum était notamment basé sur une étude préparée au préalable par deux consultants pour le compte du Vanuatu Environment Unit (Kick, Hakwa, 2002). À l'issue de ce forum, la recommandation principale de cette étude était adoptée. Celle-ci consistait à définir le mandat du conseil de la recherche scientifique comme double : protéger les intérêts du Vanuatu en termes de développement et servir d'intermédiaire pour protéger les droits des populations traditionnelles, à travers notamment la mise en place de mécanismes concrets de CIP et de règles d'Accès et de partage des avantages (APA).

Le futur Conseil devait agir comme un organisme chapeautant toute la recherche scientifique ayant lieu sur le sol du Vanuatu, à l'exclusion de la recherche médicale. 


\section{Les termes du débat au niveau international}

Les pratiques des scientifiques liées à la collecte, à l'échange ou à l'étude de matériels biologiques sont donc aujourd'hui fortement questionnées voire remises en cause sur la base d'objectifs d'équité et d'éthique reformulés par les politiques internationales liées à la CDB.

Toutefois, à l'échelle internationale, les discussions sont entièrement focalisées sur les représentants des deux extrémités de la chaîne, à savoir les communautés locales, d'une part, et les firmes multinationales, d'autre part. Il est vrai que le processus de bioprospection met de facto en relation ces deux groupes humains qui ne disposent que de peu de normes communes. Il convient néanmoins de souligner que l'échange se fait rarement directement entre eux. Une multitude d'intermédiaires interviennent entre les deux extrémités de la chaîne.

Parmi ces intermédiaires, les scientifiques, qui ont eux aussi leurs propres normes, jouent un rôle central. Russel Barsh (2001) a ainsi montré que la recherche est l'intermédiaire privilégié de collecte pour toute entreprise utilisatrice de ressources génétiques ou de savoirs traditionnels et que, parmi les sources de bioprospection des entreprises qui utilisent les savoirs traditionnels, la littérature scientifique figure au premier rang.

En tant qu'intermédiaires, aussi bien d'échantillons biologiques, que de connaissances, de technologies voire d'institutions, les chercheurs occupent indéniablement une place centrale dans la chaîne d'innovation du vivant entre populations marginalisées et acteurs économiques, entre pays riches en biodiversité et régions riches en technologies, entre acteurs locaux et acteurs globaux.

L'intervention des scientifiques dans la chaîne de valorisation revêt plusieurs formes. Ils peuvent agir en tant qu'intermédiaires directs prenant part euxmêmes, en totalité ou partiellement, à l'activité de bioprospection. Mais ils peuvent également être des intermédiaires indirects lorsqu'une collecte de ressources biologiques à des fins scientifiques ou une publication sur les savoirs traditionnels est utilisée dans un second temps à des fins commerciales.

Leur participation implique également souvent différents degrés de manipulation de la ressource originale. Ils peuvent simplement collecter, classer et stocker la ressource; ils peuvent traduire, cataloguer et publier un savoir traditionnel ; ils peuvent aussi l'utiliser en tant qu'input dans leur propre processus de recherche fondamentale ou appliquée. Dans ce cas, la ressource va être manipulée et transformée.

Kelly Bannister, Heather Mahony et R. Michael M'Gonigle (à paraitre) expliquent bien, par exemple, le processus de transformation opéré par le travail de l'ethnobotaniste. Après l'enregistrement, l'isolement et la traduction du savoir associé à une plante dans un langage et un format reconnus par le système 
scientifique international, un nouvel objet est créé sur lequel un droit de propriété peut s'attacher. Il peut alors devenir échangeable et commercialisable dans le système marchand. Le langage scientifique a donc la capacité de transformer une connaissance en un objet tangible (la propriété physique d'une connaissance portant sur une plante médicinale). En ce sens, elle constitue une « découverte » au sens de la propriété intellectuelle et facilite donc son exploitation commerciale.

Malgré ces évidences, les négociations internationales concernant la régulation des échanges de ressources génétiques restent fortement polarisées et peu attentives aux rôles et aux responsabilités des intermédiaires. Il est vrai que le secteur de la recherche scientifique (en tant qu'utilisateur et intermédiaire, et non en tant qu'expert) n'est que peu représenté dans ces enceintes et les seules voix venues de celui-ci lorsqu'il est présent consistent à défendre l'extériorité de ce secteur par rapport à la chaîne de valorisation et à réclamer une exemption de toutes règles contraignantes qui pourraient s'appliquer aux échanges et menacer la poursuite de leurs activités de recherche.

\section{CONCLUSION}

Les vicissitudes rencontrées par l'expédition Santo 2006 ne sont qu'une illustration concrète de cette absence de dialogue et de prise en compte de la place de la recherche dans les discussions relatives à la bioprospection et à la protection des savoirs traditionnels.

Il est vrai que, bien souvent, le rôle d'intermédiaire est souvent joué de manière non intentionnelle. Ainsi, interrogés sur leurs pratiques, beaucoup de chercheurs se sentent souvent peu concernés par les débats sur la régulation des échanges de ressources génétiques. S'ils conviennent subir des désagréments dans l'exercice de leur métier, leur diagnostic porte davantage sur l'absence de règles claires de la part des pays «fournisseurs » que sur l'insuffisance de règles au niveau de leurs propres institutions.

Pourtant, beaucoup d'entre eux reconnaissent que la mise en conformité avec l'esprit de la CDB nécessite une réponse plus collective qu'individuelle. Cela est devenu d'autant plus crucial qu'au sein des négociations sur le régime international d'accès et de partage des avantages, d'autres mécanismes que le Consentement préalable informé ont vu le jour. Ces mécanismes, destinés à garantir plus de transparence et de traçabilité tout au long de la chaîne de valorisation du vivant (certificats de conformité, mécanismes de respect des obligations...), vont, dans le futur, complexifier encore davantage le travail des chercheurs. Et ce malgré le « succès » obtenu par les scientifiques de se voir reconnaître, au sein de l'Article 8 du Protocole (Considérations spéciales), un traitement «simplifié » en cas de recherche non-commerciale. 
Car, indépendamment des règles fixées dans le Protocole (qui vont se retrouver ou pas dans de futures législations nationales), il est évident que plus de dix ans de discussions ont irréversiblement modifié les perceptions des différents acteurs et que la manière dont sont conduites les recherches, et les prospections naturalistes en particulier, en sera durablement modifiée.

\section{BIBLIOGRAPHIE}

Bannister K., Mahony H., M'Gonigle R. M., à paraître, "Plants and Knowledge, Power and Wealth: The Political Economy of Ethnobotany and Traditional Medicines » in Steinberg M. (dir.), Forest, Fields and Fish: Politicized Indigenous Resources, University of Texas Press, disponible sur http://www.polisproject.org/ PDFs/Bannister_et_al_Plants.pdf

Barsh R. L., 2001, "Who Steals Indigenous Knowledge? ", Proceedings $9^{\text {th }}$ Annual Meeting American Society Of International Law, pp. 153-161.

Berlin B., Berlin E. A., 2003, "Les ONG et le processus d'autorisation préalable dans la recherche de bioprospection : le projet Maya ICBG aux Chiapas, Mexique ", Revue internationale des sciences sociales, "Les ONG et la gouvernance de la biodiversité » (coord. Marie Roué), $n^{\circ}$ 178, vol. 4, pp. 689-698.

Bolton L., 1999, « Introduction », Oceania, n 1, vol. 70, pp. 1-8.

Brahy N., Louafi S. 2003. "La Convention sur la diversité biologique à la croisée de quatre discours ", Les rapports de l'lddri, n 3 .

Charvolin F., 2004, "Le programme Feederwatch et la politique des grands nombres ", Développement durable et territoires, http://developpementdurable.revues.orf/ documents687.html

CDB (Convention sur la diversité biologique), 1992, Nations Unies.

Deroche F., 2006, " L'émergence du mouvement international des peuples autochtones " in Fritz J.-C., Deroche F., Fritz G., Porteilla R. (dir.), La nouvelle question indigène, Peuples autochtones et ordre mondial, Paris, L'Harmattan, pp. 65-73.

Ducournau P., 2009, "Acteurs face à la biopolitique : Capacités critiques et tactiques de résistance ", Cahiers internationaux de sociologie, $n^{\circ} 127$, pp. 291-314.

Dumoulin D., 2003, « Les savoirs locaux dans le filet des réseaux transnationaux des ONG : perspectives mexicaines », Revue internationale des sciences sociales, "Les ONG et la gouvernance de la biodiversité » (coord. Marie Roué), $n^{\circ} 178$, vol. 4, pp. 655-666.

Faugère E., 2008, «L'exploration contemporaine de la biodiversité. Approche anthropologique de l'expédition Santo 2006 ", Journal de la société des océanistes, n 126-127, pp. 195205.

Fritz J.-C., Deroche F., Fritz G., Porteilla R. (dir.), 2005, La nouvelle question indigène. Peuples autochtones et ordre mondial, Paris, L'Harmat$\tan$.

Gray B., 1978, "Complexities of Informed Consent », Annals of the American Academy of Political and Social Science, "Medical Ethics and Social Change », vol. 437, pp. 37-48.

IDDRI (Institut du développement durable et des relations internationales), 2002, Naissance et dynamique de la question de la conservation des savoirs et savoir-faire des communautés autochtones et locales, Document préparatoire de l'atelier organisé par I'IDDRI et l'Institut français de la biodiversité : La protection des savoirs locaux, des expériences africaines et 
européennes, 11 avril 2002, La Haye, pp. 113.

Kick C. G., Hakwa M. T., 2002, A Study of the Establishment of a National Scientific Research Council for Vanuatu, Final Report to the Vanuatu Environment unit of the Ministry of Lands, février.

Miller T., Boulton M., 2007, " Changing Constructions of Informed Consent: Qualitative Research and Complex Social Worlds ", Social Science and Medicine, $n^{\circ}$ 11, vol. 65, pp. 2199-2211.

Perrin H., 2006, «Les peuples autochtones, à l'origine d'un renouveau du principe d'autodétermination ? » in Fritz J.-C., Deroche F.,
Fritz G., Porteilla R. (dir.), La nouvelle question indigène. Peuples autochtones et ordre mondial, Paris, L'Harmattan, pp. 261-273.

Posey D., Dutfield G., 1997, Le marché mondial de la propriété intellectuelle. Droits des communautés traditionnelles et indigènes, Ottawa/Genève, Centre de recherches pour le développement international/WWF.

Royson W., 2006, "Scientific Research Gets First Butting from Govt Institution », Daily Post, $\mathrm{n}^{\circ}$ 1764, 28 juin.

Tryon D., 1999, "Ni-Vanuatu Research and Researchers », Oceania, $n^{\circ} 1$, vol. 70 , pp. 9-15. 